\title{
Baicalein improves behavioral dysfunction induced by Alzheimer's disease in rats
}

This article was published in the following Dove Press journal:

Neuropsychiatric Disease and Treatment

9 December 2016

Number of times this article has been viewed

\author{
Li Zhou \\ Sha Tan \\ Yi-long Shan \\ Yu-ge Wang \\ Wei Cai \\ Xue-hong Huang \\ Xi-yuan Liao \\ Hai-yan Li \\ Lei Zhang \\ Bing-jun Zhang \\ Zheng-qi Lu
}

Department of Neurology, The Third Affiliated Hospital of Sun Yat-sen University, Guangzhou, Guangdong,

People's Republic of China
Correspondence: Zheng-qi Lu Department of Neurology, The Third Affiliated Hospital of Sun Yat-sen University, 600 Tianhe Road, Guangzhou, Guangdong 510630, People's Republic of China

Tel/fax +86203834 49l0

Email luzhengqi239@hotmail.com
Background: Alzheimer's disease (AD) is considered to be a neurodegenerative disorder that is characterized by increased oxidative stress. Medicinal plants, with their antioxidant properties, have been used to cure several human diseases. The aim of the current study was to explore the protective and therapeutic effect of baicalein on $\mathrm{AD}$-induced rats.

Materials and methods: Swiss Wistar rats were used in the study. The rats were divided into five groups. Group I: normal control group treated with water; Group II: disease control treated with $\mathrm{AlCl}_{3}$ to induce the mimicking $\mathrm{AD}$ for 4 successive weeks (SW); Group III: normal control group treated with baicalein $(5 \mathrm{mg} / \mathrm{kg})$ for $2 \mathrm{SW}$ followed by combination of baicalein and $\mathrm{AlCl}_{3}$ for $4 \mathrm{SW}$; Group IV: normal control group treated with baicalein $(10 \mathrm{mg} / \mathrm{kg})$ for $2 \mathrm{SW}$ followed by combination of baicalein and $\mathrm{AlCl}_{3}$ for $4 \mathrm{SW}$; Group V: normal control group treated with rivastigmine $(0.3 \mathrm{mg} / \mathrm{kg})$ for $2 \mathrm{SW}$ followed by combination of rivastigmine and $\mathrm{AlCl}_{3}$ for $4 \mathrm{SW}$. Moreover, the therapeutic groups are as follows: Group VI: AD disease control treated with $\mathrm{AlCl}_{3}$ for $4 \mathrm{SW}$ and serving as the therapeutic positive group; Group VII: AD disease control + baicalein $(5 \mathrm{mg} / \mathrm{kg})$ for $12 \mathrm{SW}$; Group VIII: AD disease control + baicalein $(10 \mathrm{mg} / \mathrm{kg})$ for $12 \mathrm{SW}$; Group IX: AD disease control + rivastigmine $(0.3 \mathrm{mg} / \mathrm{kg})$ for $12 \mathrm{SW}$. Behavioral test, T-maze, and rotarod test were also performed before and after the treatment. At the end of the experimental study, all the rats were sacrificed and their brains were removed and divided into two portions. The first portion was homogenated for estimating the level of acetylcholinesterase (AchE) and acetylcholine (Ach). Another portion was used for histopathological evaluation.

Results: The current investigation showed that baicalein significantly reduced the duration of revolving on the rotarod, cage activity, and T-maze activity in a dose-dependent manner compared with the AD control group rats. It also altered the AchE and Ach levels in the brain homogenates. The histopathology study also provides strength to the protective effect of baicalein.

Conclusion: The current study showed that baicalein significantly $(P<0.05)$ improved the biochemical and histopathological condition of AD in rats.

Keywords: baicalein, Alzheimer's disease, acetylcholinesterase, acetylcholine

\section{Introduction}

Alzheimer's disease (AD) is a neurodegenerative disorder that is characterized by the progressive degeneration of cortical neurons and hippocampal cells, which has a negative effect on cognitive and memory function. The first clinical sign of the disease is the deterioration of short-term memory, whereas recovery of distant memories occurs relatively late in the course of the disease. At the onset of the disease, additional cognitive capabilities are impaired, as is the capability to analyze, and use common tools and objects. Senile plaques are the pathological hallmarks of AD, which are special depositions of protein $\beta$-amyloid characterized by the formation of neurofibrillary tangles and neuronal processes, composed of paired helical filaments and various proteins. ${ }^{1,2}$ 
The selective deficiency of acetylcholine (Ach) in AD has given rise to the "cholinergic hypothesis", which proposes that deficiency of Ach is critical for the genesis of the symptoms of $\mathrm{AD}$. Therefore, a major approach to the treatment of $\mathrm{AD}$ involves attempts to augment the cholinergic function of the brain. This involves the use of cholinesterase inhibitors such as tacrine, galantamine, rivastigmine, and donepezil. Moreover, other hypotheses state that inflammation plays a key role in the pathogenesis of $\mathrm{AD}$. In addition, excessive levels of reactive oxygen species are implicated in the etiology of AD.

Baicalein (5,6,7-trihydroxy-2-phenyl-4H-1-benzopyran4-one), a flavonoid commonly known as Huangqin (China) and Ogon (Japan), is extracted from Scutellaria baicalensis. Various investigations have confirmed that baicalein has an anti-inflammatory, antidiabetic, antibacterial, antimicrobial, and anticancer effects. Moreover, its scavenging effects on various free radicals provide evidence of its antioxidant properties.

Medicinal plants have traditionally been used to treat several human diseases, and their pharmacological and therapeutic properties have been attributed to different chemical constituents isolated from their crude extracts. In particular, chemical constituents with antioxidant activity can be found at high concentrations in plants and can be responsible for their protective effects against various degenerative diseases, including cancer and neurological and cardiovascular disease. Thus, the antioxidant properties of plants have a full range of prospective applications in human health care.

\section{Materials}

Baicalein and aluminum chloride $\left(\mathrm{AlCl}_{3}\right)$ were purchased from Sigma-Aldrich Co., St Louis, MO, USA.

\section{Animals}

In the current study, we used the Swiss albino Wistar rats (male). The Wistar rats (140-190 g) were obtained from our institutional animal house. The rats were kept in a single cage in the departmental animal house under standard laboratory conditions (temperature $22^{\circ} \mathrm{C} \pm 5^{\circ} \mathrm{C}, 12$ hour dark/light cycles and artificially illuminated). The rats received a standard diet and water ab libitum during the acclimation time (1 week). This study was approved by the Sun-Yat Sen University Animal Ethical Committee and the study was performed in accordance with the committee's guidelines for animal experiments.

\section{Experimental study}

During the experiment, the rats were divided into nine groups, with each group comprising 10 rats.

\section{Protective groups}

Group I: Normal control group treated with water.

Group II: Disease control group treated with $\mathrm{AlCl}_{3}$ to induce the mimicking of $\mathrm{AD}$ for 2 successive weeks.

Group III: Normal control group treated with baicalein $(5 \mathrm{mg} / \mathrm{kg}$ ) for 2 successive weeks, followed by a combination of baicalein and $\mathrm{AlCl}_{3}$ for 4 successive weeks.

Group IV: Normal control group treated with baicalein $(10 \mathrm{mg} / \mathrm{kg}$ ) for 2 successive weeks, followed by a combination of baicalein and $\mathrm{AlCl}_{3}$ for 4 successive weeks.

Group V: Normal control group treated with rivastigmine $(0.3 \mathrm{mg} / \mathrm{kg})$ for 2 successive weeks, followed by a combination of rivastigmine and $\mathrm{AlCl}_{3}$ for 4 successive weeks.

\section{Therapeutic groups}

Group VI: AD disease control group treated with $\mathrm{AlCl}_{3}$ for 4 successive weeks and serving as the therapeutic positive group.

Group VII: AD disease control group + baicalein $(5 \mathrm{mg} / \mathrm{kg})$ for 12 successive weeks.

Group VIII: AD disease control + baicalein $(10 \mathrm{mg} / \mathrm{kg})$ for 12 successive weeks.

Group IX: AD disease control + rivastigmine $(0.3 \mathrm{mg} / \mathrm{kg})$ for 12 successive weeks.

\section{Behavioral stress tests}

In the behavioral stress tests (the rotarod test and the cage test), the percentage behavior change was estimated using a square root transformation. This calculation was used to mitigate against the normal biological variations of the control rats in each group. Later, the square root transformed changes for each rat were compared with the baseline activity at each transitional step for the entire experimental study.

\section{Estimation of motor coordination using the rotarod test}

In the current investigation, the level of motor coordination was appraised using the accelerating rotarod test according to the method described by Mahdy et al, ${ }^{3}$ with minor modification.

\section{Estimation of cognitive capabilities using T-maze test}

The T-maze test was used to estimate the cognitive capability of the rats according to the method described by Deacon and Rawlins, with minor modification. ${ }^{4}$ 


\section{Cage test}

The cage test was used to estimate the movement of the rats according to the method described by Tsakiris et al, ${ }^{5}$ with minor modification.

\section{Brain tissue preparation}

At the end of the experimental study, all the rats were fasted overnight and killed by decapitation. For each group, the rat brain was immediately removed, washed in isotonic saline, and weighed. Next, the rat brain was divided into two portions. The first brain portion of each rat was homogenized in ice-cold medium $(300 \mathrm{mmol} / \mathrm{L}$ sucrose and $50 \mathrm{mmol} / \mathrm{L}$ Tris-Hcl $[\mathrm{pH}=7.4])$. The homogenate was centrifuged at $5,000 \mathrm{rpm}$ for 5 minutes at $25^{\circ} \mathrm{C}$. The collected supernatant was used to estimate biochemical parameters such as total protein, acetylcholinesterase (AchE), and Ach. The other portion of brain tissue was fixed in 10\% formalin for histopathological observation.

\section{Biochemical estimation}

A choline/Ach assay kit was used to estimate the level of brain Ach, according to the manufacturer's instructions. A colorimetric method was used to estimate the level of brain AchE. The brain protein concentration was determined (to express the level of protein in the brain) using the method described by Lowry et al. ${ }^{6}$

\section{Western blot analysis}

Using western blot analysis, cortical and hippocampal tissues were homogenized using ice-cold buffer. All the tissues were homogenized by high-speed centrifugation at $12,000 \mathrm{rpm}$ for 20 minutes. The $50 \mu \mathrm{g} / \mathrm{mL}$ protein samples underwent sodium dodecyl sulfate $(10 \%)$ polyacrylamide gel electrophoresis, after which the gel was transferred to polyvinylidene difluoride membrane. The prepared membrane was then incubated for 2 hours to remove the nonspecific binding site. Next, different proteins - namely, $\beta$-actin, $\gamma$ and $\beta$ secretases, $\beta$-amyloid, and amyloid precursor protein (APP) - were used.

\section{Histopathological examination}

The second portion of the rat brain from each group was fixed in buffer formalin (10\%) for 24 hours. The brain tissue was washed in tap water and then dehydrated using serial dilutions of alcohol (ethyl, methyl, and absolute ethyl). The prepared tissue was cleared with xylene and embedded in paraffin for 24 hours, then a microtome was used to cut paraffin bees wax tissue blocks. The prepared tissue sample was placed on a glass plate, deparaffinized, and stained with eosin and

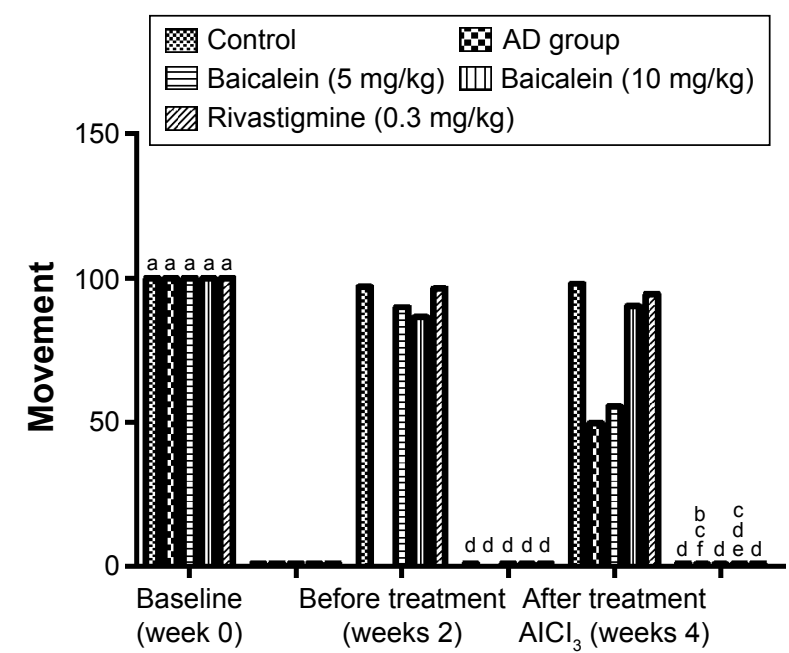

Figure I Protective effects of baicalein and rivastigmine on the levels of activity in AD-induced rats in activity cages.

Notes: All data are expressed as mean of movements \pm SE. $\%$ change; bsquare root transformed \% change; 'significantly different from baseline of the same group at $P<0.05$; ${ }^{d}$ significantly different from $A D$ group at $P<0.05$; ${ }^{\mathrm{e}}$ significantly different from rivastigmine when each is used for 4 weeks in combination with $\mathrm{AlCl}_{3}$ at $P<0.05$; ${ }^{f}$ significantly different from same group when given alone for 2 weeks at $P<0.05$. Abbreviations: AD, Alzheimer's disease; SE, standard error.

hematoxylin strains for histopathological observation under a light microscope.

\section{Results \\ Protective effect}

\section{Cage activity}

Figure 1 shows the significant reduction in cage activity of the rats treated with $\mathrm{AlCl}_{3}$ after 4 weeks, compared with the activity of the baseline group of the same group. The $\mathrm{AlCl}_{3}$ control group rats treated with baicalein and rivastigmine showed increased activity in a dose-dependent manner. Baicalein $(5 \mathrm{mg} / \mathrm{kg})$ group rats showed an increase in cage activity compared with the $\mathrm{AlCl}_{3}$ group rats. Baicalein $(10 \mathrm{mg} / \mathrm{kg})$ group rats showed a more marked effect. However, the rivastigmine group rats showed marked improvement in activity compared with the $\mathrm{AlCl}_{3}$ group.

\section{Effect on T-maze activity}

The T-maze test result showed a significant increase in the time taken by the $\mathrm{AlCl}_{3}$ group rats to reach the food over 4 successive weeks. Figure 2 shows that the $\mathrm{AlCl}_{3}$ group rats treated with baicalein and rivastigmine showed a reduction in the time taken by the rats to reach the food compared with the time taken by the $\mathrm{AD}$ group rats.

\section{Effect on rotarod activity}

Figure 3 shows that the $\mathrm{AD}$ group rats treated with baicalein and rivastigmine showed no significant change in motor coordination in the rotarod test when compared with the baseline 


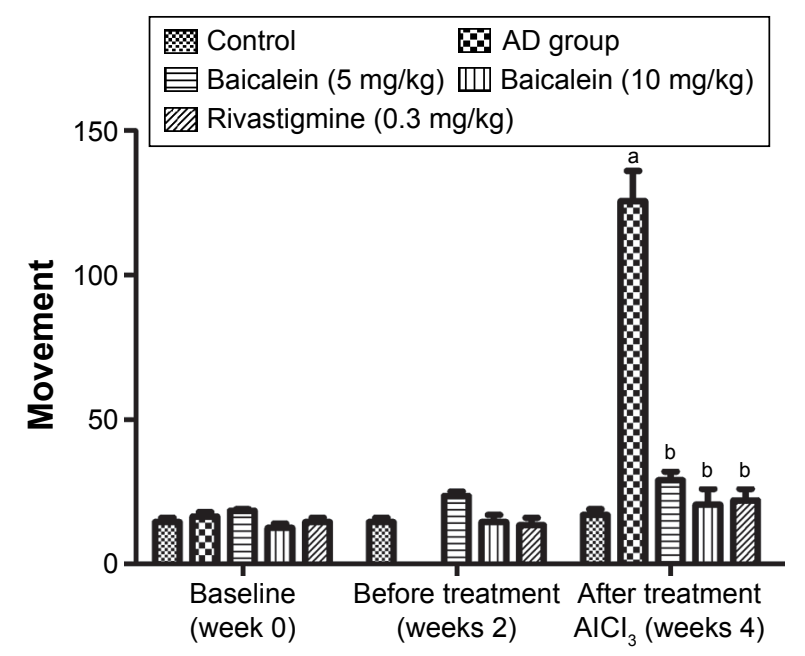

Figure 2 Protective effects of baicalein and rivastigmine on the time taken to find the food in the T-maze by AD-induced rats.

Notes: All data are expressed in seconds as mean \pm SE. ${ }^{a}$ Significantly different baseline duration of the same group at $P<0.05$; bsignificantly different from $\mathrm{AlCl}_{3}$ after 4 weeks induction at $P<0.05$.

Abbreviations: AD, Alzheimer's disease; SE, standard error.

value groups. The result demonstrated that there were no significant changes in duration of sustained balance for the rats over 4 successive weeks.

\section{Biochemical parameters}

Figure 4 shows a significant reduction in the Ach levels found, and also confirms the enhanced level of AchE in the brain of the rats treated with $\mathrm{AlCl}_{3}$ compared with those in the normal control group rats over 4 successive weeks. $\mathrm{AlCl}_{3}$-treated rats that received baicalein showed a significant improvement in the level of Ach, and a reduction in the level of AchE, in a dose-dependent manner. However,

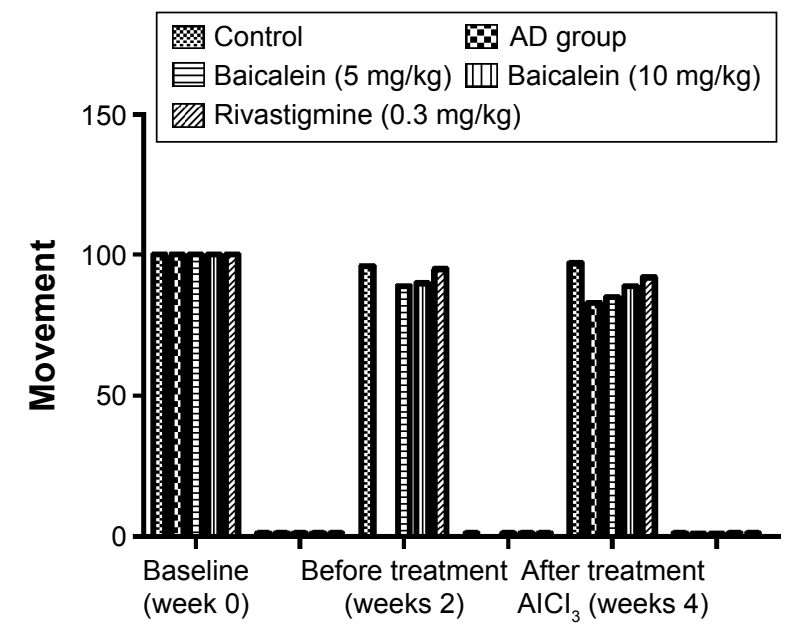

Figure 3 Protective effects of baicalein and rivastigmine on the time spent on the rotarod by AD-induced rats.

Abbreviation: AD, Alzheimer's disease. rivastigmine showed a remarkable enhancement in the level of Ach, and reduction in the level of AchE, compared with the $\mathrm{AD}$ control group rats.

\section{Therapeutic effects \\ Cage activity}

Figure 5 shows the effect of baicalein and rivastigmine on the AD-induced rats. The administration of baicalein ( 5 and $10 \mathrm{mg} / \mathrm{kg}$ ) resulted in no significant change in activity compared with the baseline activity of the same group, but baicalein enhanced cage activity compared with the same group before treatment. However, the administration of rivastigmine also showed a significant increase in cage activity.

\section{Rotarod activity}

Figure 6 shows that motor coordination activity was significantly reduced in the $\mathrm{AD}$ group rats, as tested by the rotarod, when the rats were treated with $\mathrm{AlCl}_{3}$ for 4 successive weeks. The $\mathrm{AD}$ group rats treated with the baicalein underwent a significant improvement in motor coordination activity in a dose-dependent manner.

\section{T-maze activity}

In this test, the $\mathrm{AlCl}_{3}$ ( $\mathrm{AD}$ control) group rats showed a significant increase in the duration of time to reach the food over 4 successive weeks. The rats were left for 12 weeks without treatment and also treated with baicalein and rivastigmine. The $\mathrm{AD}$ rats treated with baicalein and rivastigmine showed a significant reduction in the time taken to reach the food in the T-maze test (Figure 7).

\section{Biochemical parameters}

Figure 8 shows the significant diminution in the Ach levels and also confirms the augmented level of AchE in the brain of the rats treated with the $\mathrm{AlCl}_{3}$ compared with the normal control group rats. The $\mathrm{AlCl}_{3}$-treated rats that received baicalein showed a significant improvement in the level of Ach, and reduction in the level of AchE, in a dose-dependent manner. However, rivastigmine showed a remarkable enhancement in the level of Ach, and reduction in the level of AchE, compared with the $\mathrm{AD}$ control group rats.

\section{Estimation of protein expression}

Figure 9 represents the effect of protein expression with different proteins - namely, A $\beta 1-42$, APP, $\beta$ and $\gamma$ secretases. We analyzed the effect of baicalein on the $\beta$-amyloid metabolic marker in the AD-induced rats. 

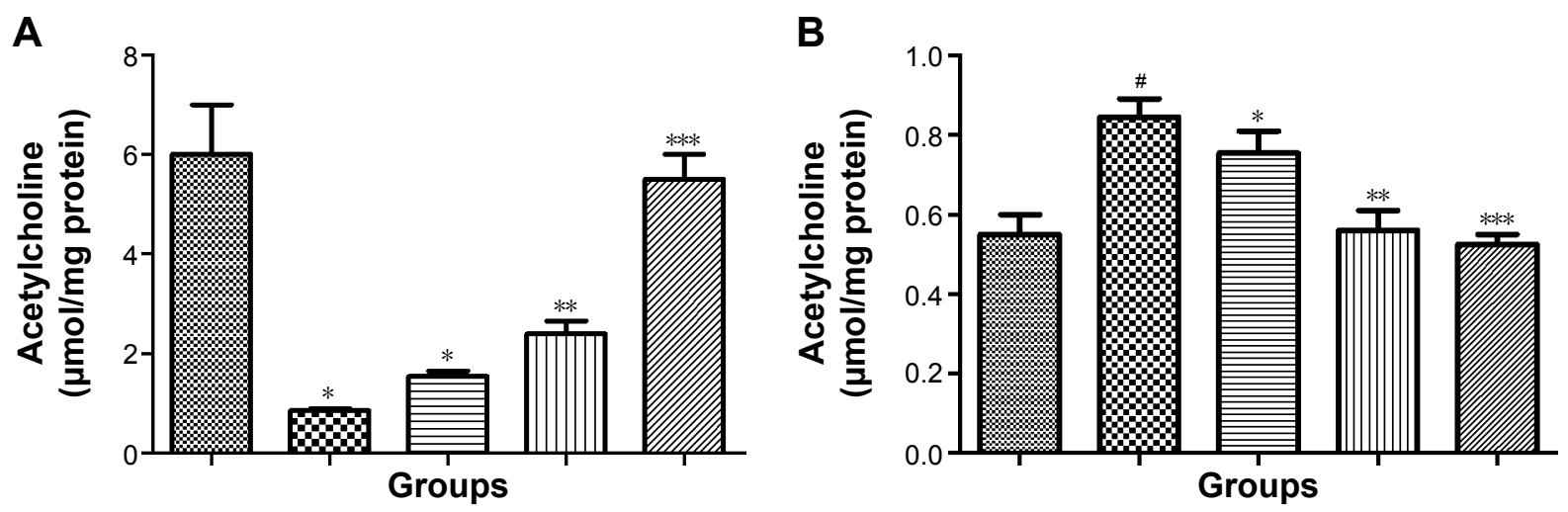

Control $\mathbf{B}$ AD group 目 Baicalein $(5 \mathrm{mg} / \mathrm{kg})$ 血 Baicalein $(10 \mathrm{mg} / \mathrm{kg})$ Rivastigmine $(0.3 \mathrm{mg} / \mathrm{kg})$

Figure 4 Protective effects of baicalein and rivastigmine on brain levels of $(\mathbf{A})$ : acetylcholine and (B): acetylcholinesterase in $A D$-induced rats.

Notes: All data are expressed as mean \pm SE. ${ }^{\sharp P}<0.05$ compared with the control group; $* P<0.05, * * P<0.01$, and $* * * P<0.00$ I compared with the $A D$ control group rats.

Abbreviations: AD, Alzheimer's disease; SE, standard error.

Histopathological results for the protective and therapeutic groups

Figure 10 shows the highly active nerve cells along with the huge nuclei in the negative control group rats. However, the $\mathrm{AD}$ control group rats showed a spongy appearance, brain necrosis, loss of structure, plaques, nuclei of cells, and outlines. Some of the nuclei showed a ring appearance. AD group rats treated with baicalein and rivastigmine showed an improvement in histopathological changes compared with the AD control.

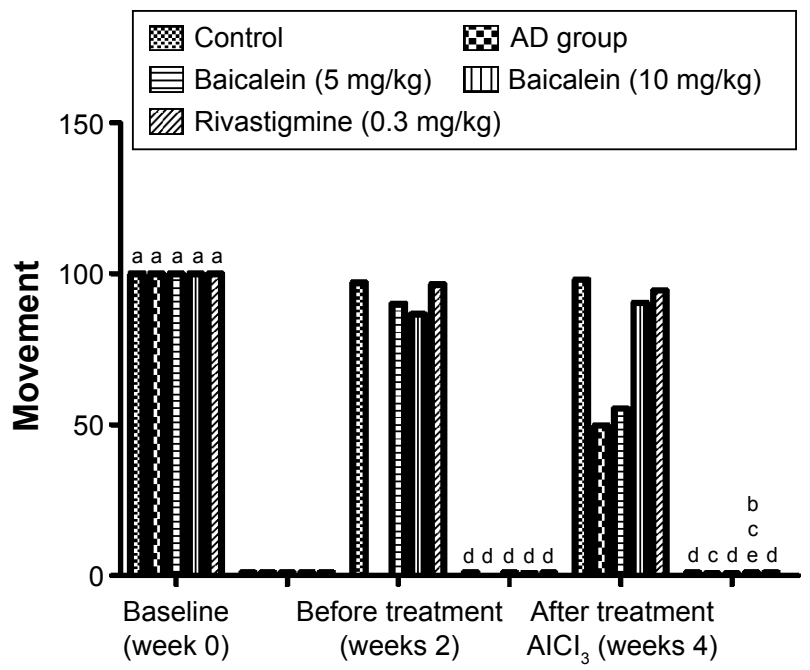

Figure 5 Protective effects of baicalein and rivastigmine on the levels of activity in AD-induced rats in activity cages.

Notes: All data are expressed as mean of movements \pm SE. a\% change; bsquare root transformed \% change; 'significantly different from baseline of the same group at $P<0.05$; ${ }^{d}$ significantly different from $A D$ group at $P<0.05$; ${ }^{e}$ significantly different from same group when given alone for 2 weeks at $P<0.05$.

Abbreviations: AD, Alzheimer's disease; SE, standard error.

\section{Discussion}

$\mathrm{AD}$ is considered to be a neurodegenerative disorder differentiated by progressive degeneration of the cortical neurons and hippocampal cells, which negatively affects the cognitive ability and memory function. AD is commonly considered to be a precursor dementia. AD complications increase with age-related changes. A decline in short-term memory is

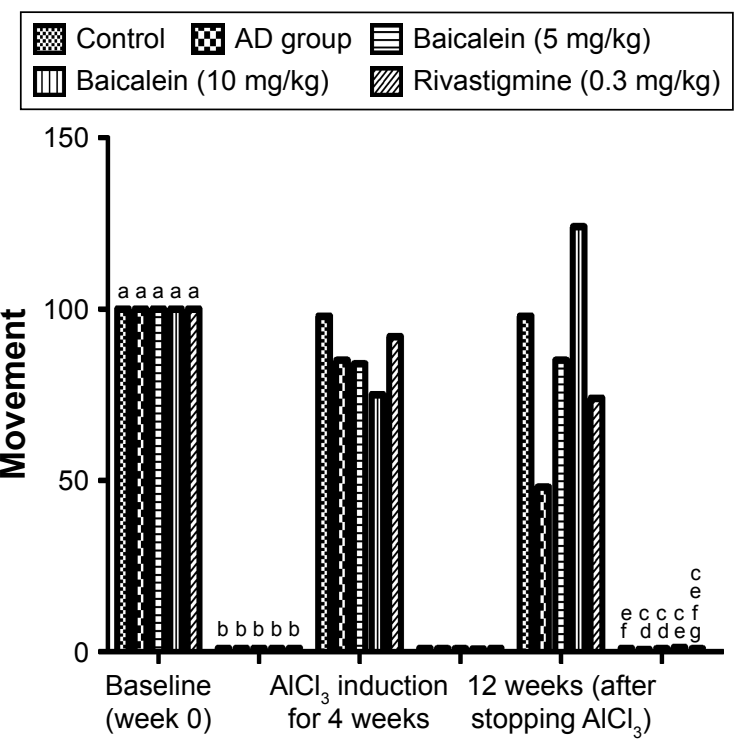

Figure 6 Protective effects of baicalein and rivastigmine on the time taken to find the food in the T-maze by AD-induced rats.

Notes: All data are expressed in seconds as mean \pm SE. aSignificantly different baseline duration of the same group at $P<0.05$; bsignificantly different from $\mathrm{AlCl}_{3}$ after 4 weeks induction at $P<0.05$; 'significantly different from baseline of the same group

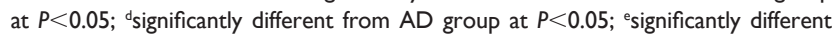
from rivastigmine when each is used for 4 weeks in combination with $\mathrm{AlCl}_{3}$ at $P<0.05$; 'significantly different from same group when given alone for 4 weeks at $P<0.05$; ${ }^{\circ}$ compared with the baicalein $(10 \mathrm{mg} / \mathrm{kg})$.

Abbreviations: AD, Alzheimer's disease; SE, standard error. 


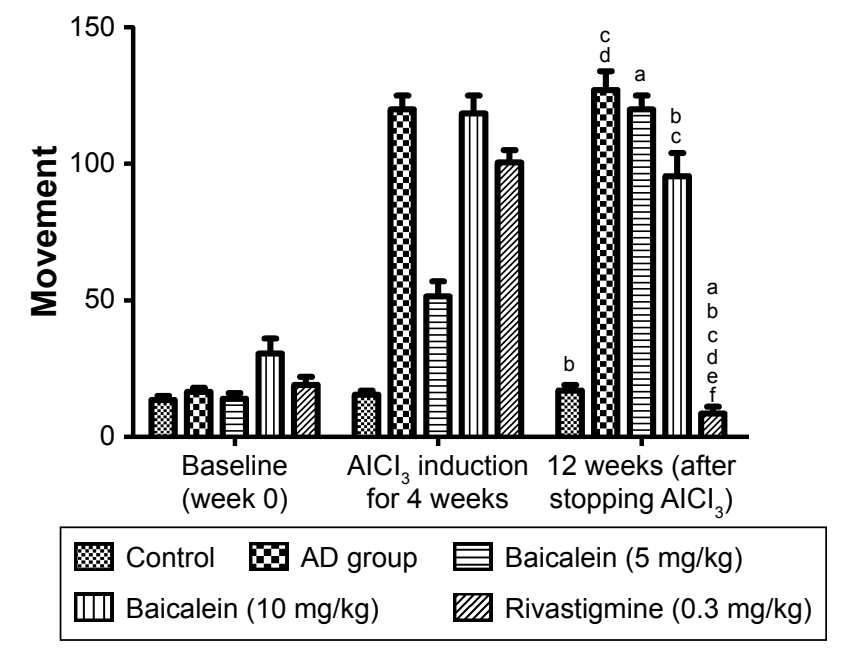

Figure 7 Protective effects of baicalein and rivastigmine on the time spent on the rotarod by AD-induced rats.

Notes: aSignificantly different baseline duration of the same group at $P<0.05$; bsignificantly different from $\mathrm{AlCl}_{3}$ after 4 weeks induction at $P<0.05$; 'significantly different from baseline of the same group at $P<0.05$; ${ }^{\circ}$ significantly different from AD group at $P<0.05$; 'esignificantly different from rivastigmine when each is used for 4 weeks in combination with $\mathrm{AlCl}_{3}$ at $P<0.05$; fsignificantly different from same group when given alone for 4 weeks at $P<0.05$.

Abbreviation: AD, Alzheimer's disease.

commonly considered to be the first clinical sign of AD; thus, the level of cognitive ability, along with the capacity to use common tools and objects, is used to estimate the progression of short-term memory. ${ }^{7,8}$ Anders and Martin reported that 35.6 million people have dementia worldwide, and this figure is predicted to rise to $65.7-115.4$ million people from 2013 to 2050. AD affects individuals of both low- and middle-income groups. Several other factors - such as deficiency of Ach, the development of atherosclerotic lesions, oxidative stress, and inflammation of the brain - also play an important role in the progression of $\mathrm{AD}$.
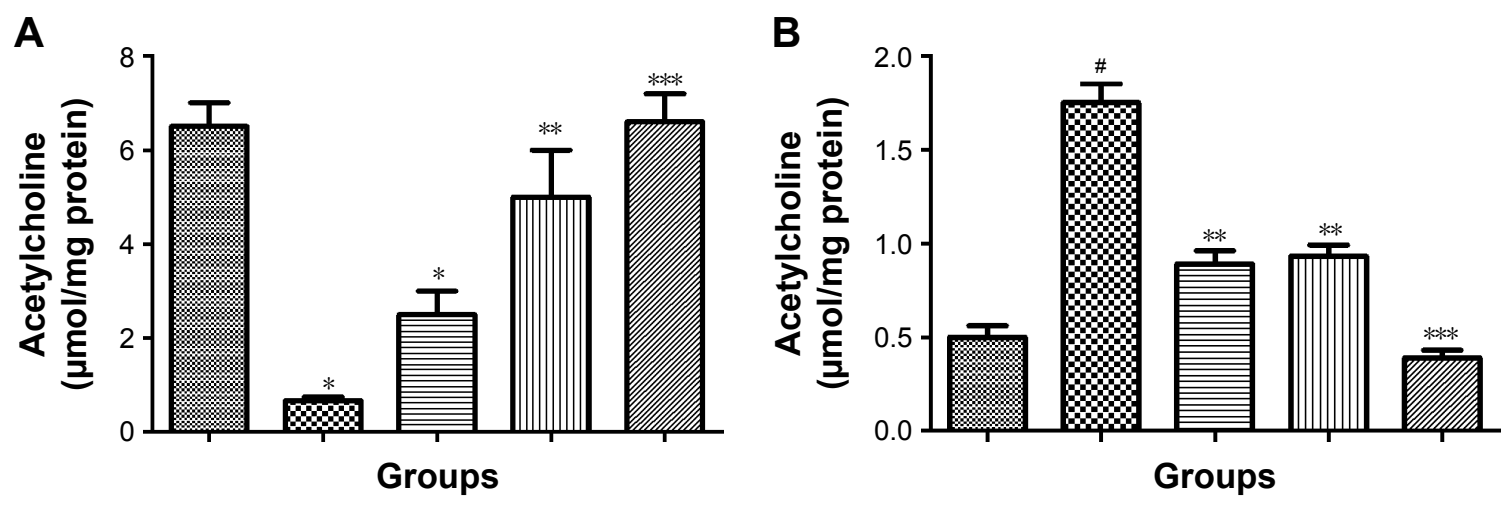

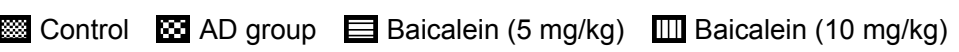

Aluminum is commonly found in soil, water, toothpaste, and food supplements, and is also used to make cooking implements. Several reports have revealed that aluminum causes the generation of free radicals in the brain and induces neurotoxicity. The deposition of free radicals in the brain may induce the degenerative events associated with aging, including $\mathrm{AD}$. In the current investigation, the $\mathrm{AD}$ rat groups treated with $\mathrm{AlCl}_{3}$ showed a reduction in cage activity and a reduced duration of rotation in the rotarod test, along with an increase in the time taken for the rats to reach the food in the T-maze test. ${ }^{910}$ An increased level of AchE, but a reduced level of Ach, was found in the disease control group rats compared with the levels found in the normal control group rats. Mayeux and Sano showed that rivastigmine is the approved drug for the treatment of $\mathrm{AD} .{ }^{11}$ It is possible that the effect of the rivastigmine on $\mathrm{AD}$ was to improve the endogenous antioxidant mechanism and the level of oxidative stress, as revealed by the improvement in cage activity; it also altered the level of Ach and AchE in the brain compared with the levels in the AD control group rats. Onor et al reported that rivastigmine enhanced both the daily living activity, and cognitive and global functions. ${ }^{12}$

The Food and Drug Administration has approved the use of AchE inhibitors to treat $\mathrm{AD}$. In the current investigation, we attempted to discover a potential new drug that could be used to prevent or slow down the disease process. Therefore, it is vital to develop herb- and medicinal plant-based drug therapies to prevent the development of neurodegenerative disorders, particularly those associated with memory dysfunction. In the current investigation, we explored the therapeutic and protective effect of baicalein vs rivastigmine

Baicalein (10 mg/kg) Rivastigmine $(0.3 \mathrm{mg} / \mathrm{kg})$

Figure 8 Protective effects of baicalein and rivastigmine on brain levels of $(\mathbf{A})$ acetylcholine and $(\mathbf{B})$ acetylcholinesterase in $A D$-induced rats.

Notes: All data are expressed as mean $\pm S E$. ${ }^{*} P<0.05$ compared with the control group; $* P<0.05, * * P<0.01$, and $* * * P<0.00$ I compared with the $A D$ control group rats. Abbreviations: AD, Alzheimer's disease; SE, standard error. 


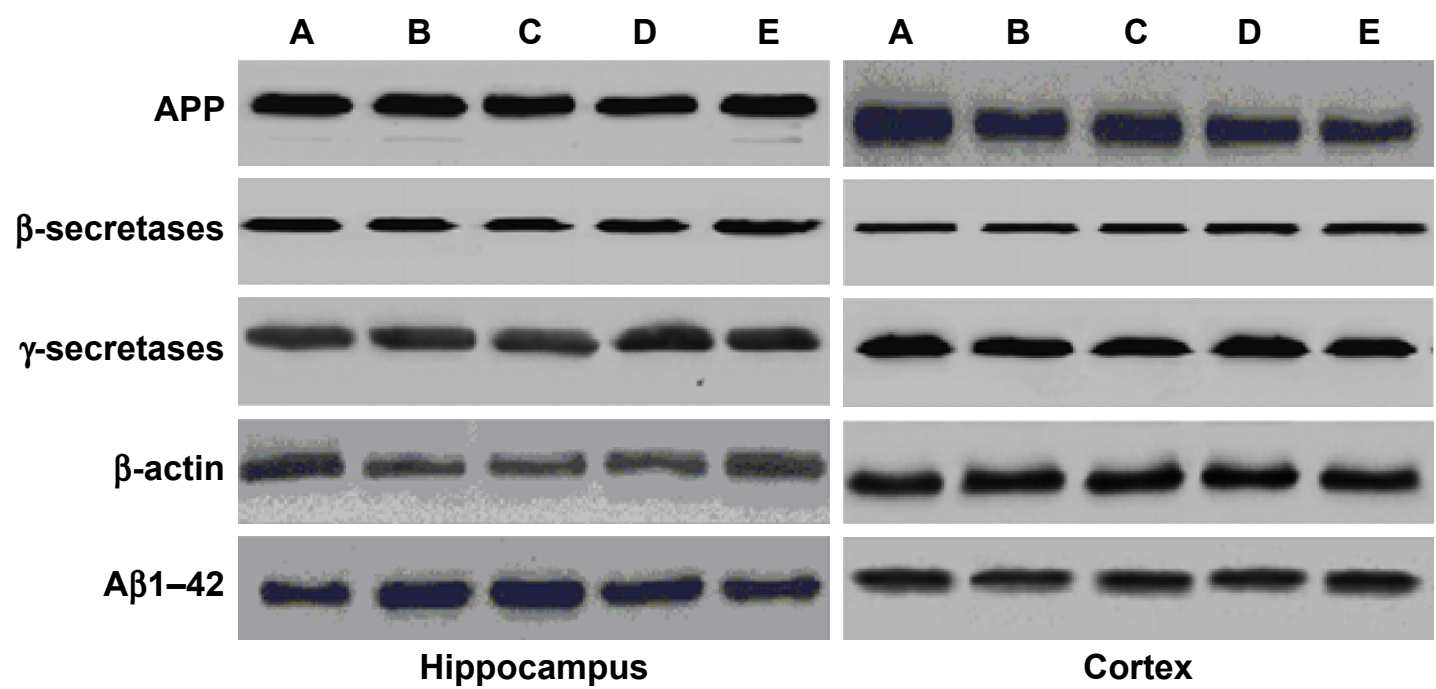

Figure 9 The expression of APP, $\beta$-secretases, $\gamma$-secretases, $\beta$-actin, and A $\beta \mid-42$ compared to control.

Notes: Groups: A: Normal; B: AD; C: AD + baicalein (5 mg/kg); D: AD + baicalein (10 mg/kg); and E: AD + rivastigmine $(0.3 \mathrm{mg} / \mathrm{kg})$.

Abbreviations: AD, Alzheimer's disease; APP, amyloid precursor protein.

in $\mathrm{AD}$ by assessing the effect of these drugs on the behavioral activity of rats. ${ }^{9,10}$

In the current investigation, we used $\mathrm{AlCl}_{3}$ to induce $\mathrm{AD}$, as revealed by a weakening in the physical state of the rats during the cage activity test, deterioration in cognitive capacity during the T-maze test, and deterioration in motor coordination during the rotarod test. It also reduced the level of Ach, and enhanced the level of AchE, in the brain homogenates of the rats. This particular effect was confirmed via a histopathological study of the rats, which revealed the existence of amyloid plaques. The AD control group rats treated with baicalein and rivastigmine confirm the therapeutic and protective effects of baicalein and rivastigmine, as shown by the improvement of behavioral status revealed by the physical state activity, time duration of motor coordination, and altered levels of activity associated with changes in Ach
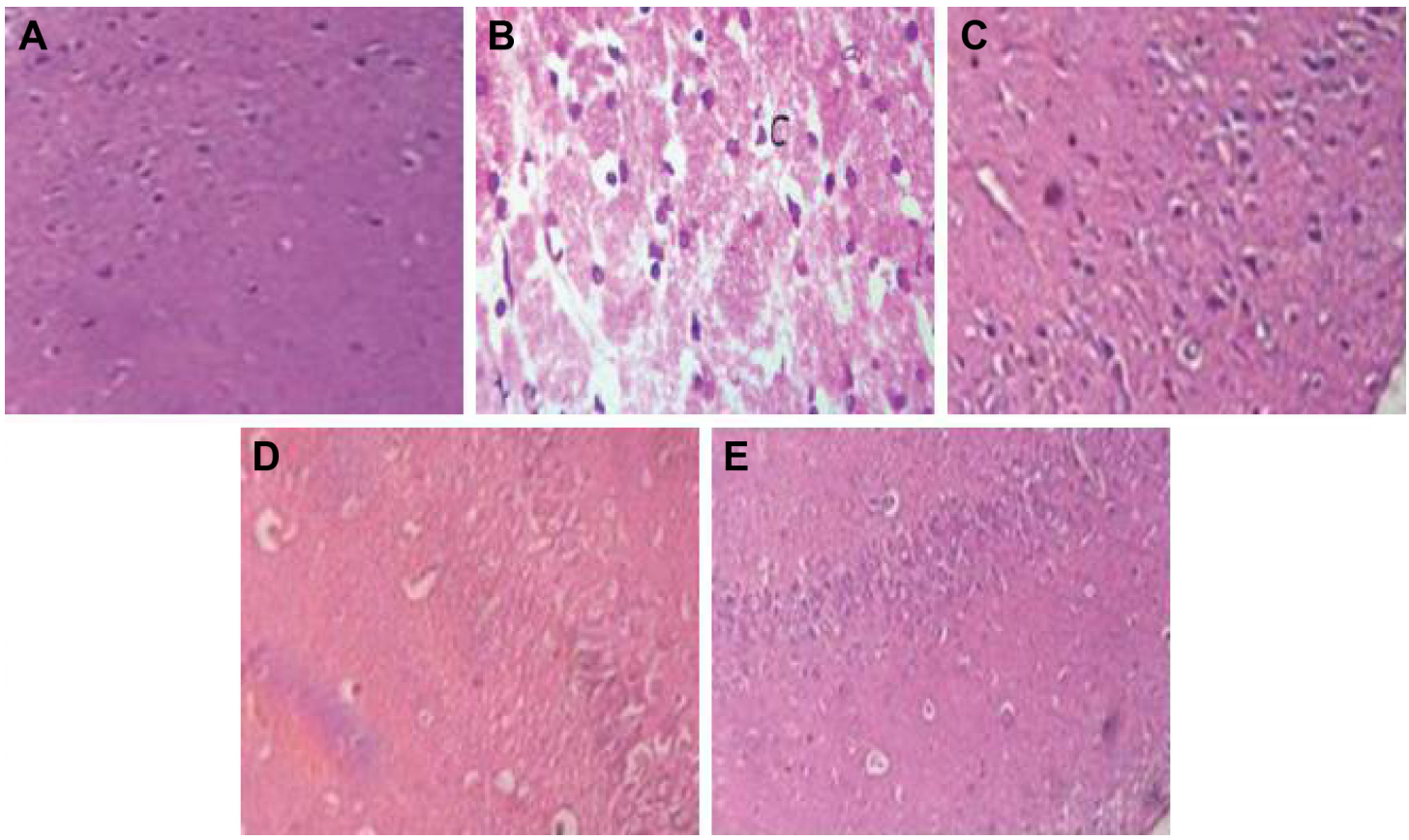

Figure 10 (A) Image of the brain section of a control rat showing normal histological structure of the hippocampus. (B) Image of the brain section of an Alzheimer's diseaseinduced rat's plaques formation. (C) AD group treated with baicalein $(5 \mathrm{mg} / \mathrm{kg})$. (D) AD group treated with baicalein $(10 \mathrm{mg} / \mathrm{kg})$. (E) AD group treated with rivastigmine $(0.3 \mathrm{mg} / \mathrm{kg})$. The tangle appears as a long pink filament in the cytoplasm (hematoxylin and eosin, I0×).

Abbreviation: AD, Alzheimer's disease. 
and AchE levels. This result has been further confirmed by a histopathological study that showed the disappearance of the amyloid plaques, which were induced by $\mathrm{AlCl}_{3} \cdot{ }^{13,14}$ Xiao et al and Shah and Reichman showed the possible mechanism of action of rivastigmine to be acting on the glutamate mechanisms, thereby reducing the oxidative stress and improving the endogenous antioxidant defense to protect against the $A \beta$-induced oxidative stress. ${ }^{15,16}$

\section{Conclusion}

In the current investigation, baicalein was shown to have a therapeutic and protective effect on the AD-induced rats. These results could be of great significance in eliminating the neurodegenerative signs of AD. Maximum effect was revealed by higher doses of baicalein. The data suggest that baicalein offers a protective and therapeutic effect against the progressive neurological injury associated with AD, particularly in relation to oxidative stress. Further clinical and molecular research is required to determine the true efficacy of baicalein on neurodegenerative disorders.

\section{Acknowledgments}

This study was supported by the Natural Science Foundation of China (number 81271328) and the Science and Technology Projects Foundation of Guangzhou City (number 2014Y2-00501).

\section{Disclosure}

The authors report no conflicts of interest in this work.

\section{References}

1. Kimura R, Ohno M. Impairments in remote memory stabilization precede hippocampal synaptic and cognitive failures in 5XFAD Alzheimer mouse model. Neurobiol Dis. 2009;33(2):229-235.
2. Yassina NAZ, El-Shenawya MA, Mahdyb KA, et al. Effect of Boswellia serrata on Alzheimer's disease induced in rats. J Arab Soc Med Res. 2013; $8: 1-11$.

3. Mahdy KA, Gouda NAM, Marrie AEH, et al. Protective effect of ginger (Zingiber officinale) on Alzheimer's disease induced in rats. $J$ Neuroinfect Dis. 2014;5:2.

4. Deacon RM, Rawlins JN. T-maze alternation in the rodent. Nat Protoc. 2006;1(1):7-12.

5. Tsakiris S, Schulpis KH, Marinou K, Behrakis P. Protective effect of L-cysteine and glutathione on the modulated suckling rat brain $\mathrm{Na}+$, $\mathrm{K}+$, -ATPase and $\mathrm{Mg} 2+-\mathrm{ATPase}$ activities induced by the in vitro galactosaemia. Pharmacol Res. 2004;49(5):475-479.

6. Lowry OH, Rosebrough NJ, Farr AL, Randall RJ. Protein measurement with the Folin phenol reagent. J Biol Chem. 1951;193(1):265-275.

7. Querfurth HW, LaFerla FM. Alzheimer's disease. N Engl J Med. 2010; 362:329-344.

8. Alzheimer's Association. 2013 Alzheimer's disease facts and figures. Alzheimer's Dement. 2013;9(2):110-133.

9. Shoji H, Hagihara H, Takao K, Hattori S, Miyakawa T. T-maze Forced Alternation and Left-right Discrimination Tasks for Assessing Working and Reference Memory in Mice. Journal of Visualized Experiments. 2012;60:1-7.

10. Hassaan Y, Handoussa H, El-Khatib AH, Linscheid MW, El Sayed N, Ayoub N. Evaluation of plant phenolic metabolites as a source of Alzheimer's drug leads. Biomed Res Int. 2014;2014:843263.

11. Mayeux R, Sano M. Treatment of Alzheimer's disease. N Engl J Med. 1999;341(22):1670-1679.

12. Onor M, Trevisiol M, Aguglia E. Rivastigmine in the treatment of Alzheimer's disease: an update. Clin Interv Aging. 2007;2(1):17-32.

13. Yassa MA. Ground zero in Alzheimer's disease. Nat Neurosci. 2014; 17:146-147.

14. Haes AJ, Chang L, Klein WL, Van Duyne RP. Detection of a biomarker for Alzheimer's disease from synthetic and clinical samples using a nanoscale optical biosensor. J Am Chem Soc. 2005;127(7):2264-2271.

15. Xiao XQ, Wang R, Han YF, Tang XC. Protective effects of huperzine A on beta-amyloid (25-35) induced oxidative injury in rat pheochromocytoma cells. Neurosci Lett. 2000;286(3):155-158.

16. Shah S, Reichman WE. Treatment of Alzheimer's disease across the spectrum of severity. Clin Interv Aging. 2006;1(2):131-142.
Neuropsychiatric Disease and Treatment

\section{Publish your work in this journal}

Neuropsychiatric Disease and Treatment is an international, peerreviewed journal of clinical therapeutics and pharmacology focusing on concise rapid reporting of clinical or pre-clinical studies on a range of neuropsychiatric and neurological disorders. This journal is indexed on PubMed Central, the 'PsycINFO' database and CAS,

\section{Dovepress}

and is the official journal of The International Neuropsychiatric Association (INA). The manuscript management system is completely online and includes a very quick and fair peer-review system, which is all easy to use. Visit http://www.dovepress.com/testimonials.php to read real quotes from published authors. 